\title{
Optical Coherence Tomography Examination of the Anterior Segment in a Case of Corneal Perforation and Lens Trauma by Chestnut Burr
}

\author{
Takashi Ono $^{\mathrm{a}}$ Yosai Mori ${ }^{\mathrm{a}} \quad$ Ryohei Nejima $^{\mathrm{a}} \quad$ Takuya Iwasaki $^{\mathrm{a}}$ \\ Shiro Amano ${ }^{\mathrm{b}}$ Kazunori Miyata ${ }^{\mathrm{a}}$ \\ ${ }^{a}$ Miyata Eye Hospital, Miyazaki, Japan; ${ }^{\text {In }}$ Inouye Eye Hospital, Tokyo, Japan
}

\section{Keywords}

Chestnut · Corneal perforation · Optical coherence tomography · Bacterial keratitis · Trauma

\begin{abstract}
Chestnut burrs, the thorny encapsulation of chestnut fruit, can sometimes cause corneal injuries and ulceration, with poor prognoses. We report a case of corneal perforation and damaged anterior lens capsule due to a chestnut burr, using anterior segment optical coherence tomography (AS-OCT). A 67-year-old woman with a chestnut burr injury in her right eye was referred to our hospital. Her right best-corrected visual acuity (BCVA) was 0.8. Slit-lamp examination and AS-OCT showed perforation involving the endothelial layer at the center of the cornea. The iris and anterior lens capsule were damaged. Cell infiltration was observed around the wound. Bacterial examination showed gram-positive cocci but no fungi. The patient was diagnosed with a corneal perforation and bacterial keratitis. Levofloxacin $1.5 \%$ and cefmenoxime treatments were initiated and a soft contact lens was placed to seal the wound. On day 3, there was no improvement in the corneal cell infiltration, but AS-OCT suggested that the inner wound had closed. A culture test revealed the presence of Propionibacterium acnes, which was sensitive to both levofloxacin and cefmenoxime. Therefore, we continued the same antibiotic treatment. On day 26 , the opacification and cell infiltration at the center
\end{abstract}


Case Reports in
Ophthalmology

Case Rep Ophthalmol 2018;9:154-159

DOI: $10.1159 / 000487076$

C 2018 The Author(s). Published by S. Karger AG, Basel www.karger.com/cop

Ono et al:: Optical Coherence Tomography Examination of the Anterior Segment in a Case of Corneal Perforation and Lens Trauma by Chestnut Burr

of the cornea had improved. AS-OCT showed healing of the corneal wound with reduction in the central corneal thickness. Her BCVA improved to 1.0. AS-OCT was a valuable tool to noninvasively observe wound shape and detect the presence of any intracorneal foreign bodies.

(c) 2018 The Author(s)

Published by S. Karger AG, Basel

\section{Introduction}

Chestnut burrs, the thorny encapsulation of chestnut fruit, sometimes cause ocular injuries in Asian countries because chestnuts are common food in Asia. The cornea, conjunctiva, and eyelid are the chief sites of injury in the eye. A previous study reported that early diagnosis and treatment are important to prevent serious complications after chestnut burr injury [1]. Small corneal and conjunctival epithelial defects are usually managed conservatively. However, progression to corneal ulceration may be associated with a poor prognosis. While chestnut burr injury is relatively common in Japan, injury to the cornea has surprisingly not been reported [1, 2]. Needles of the burr are fragile and possibly disintegrate after perforation. An earlier study has suggested that chestnut burr spines are usually well tolerated and extrude spontaneously [3]. Chemical constituents of the needles, such as saponin, may have anti-inflammatory effects [1]. However, because the needle may result in severe infection and inflammation leading to endophthalmitis, surgical removal is recommended when it is present in the eye.

We report a case of corneal perforation and damaged anterior lens capsule due to a chestnut burr, in which anterior segment optical coherence tomography (AS-OCT) was used.

\section{Case}

A 67-year-old woman reported an accidental injury to the right eye from a falling chestnut. She visited an eye clinic nearby, and was treated with ofloxacin ointment. On the next day, cell infiltration was observed around the corneal wound, suggestive of deterioration of the corneal infection. Therefore, she was referred to our hospital.

The patient complained of mild pain. At first visit, her best-corrected visual acuity (BCVA) was 0.8 in the right eye and 1.5 in the left eye. Slit-lamp examination showed a perforation at the center of the right cornea; leakage of aqueous humor through the perforation was observed (Fig. 1a). The Seidel test, which evaluates aqueous leakage on the cornea using fluorescein, was positive, although the anterior chamber was deep (Fig. 1b). Cell infiltration was observed around the wound with conjunctival injection, and moderate cells were observed in the anterior chamber. No foreign body was found in the conjunctival sac, cornea, or anterior chamber. The iris was damaged, and the anterior lens capsule was opacified with pigments (Fig. 1c, d). AS-OCT showed a corneal wound involving the endothelial layer (Fig. $1 \mathrm{e})$. The central corneal thickness was $603 \mu \mathrm{m}$. Additionally, slight cataract and early pterygium were observed. A gonioscopic examination showed no hemorrhage or foreign body at the angle of the eye. No fundus abnormality was observed. The patient was in good general health with no significant past medical history. Bacterial examination of corneal scrapings showed gram-positive cocci and a few neutrophils, but no fungi.

The patient was diagnosed with a corneal perforation and bacterial keratitis. Levofloxacin $1.5 \%$ and cefmenoxime administration every 2 hours, atropine administration once a 
day, and oral acetazolamide were initiated. A soft contact lens that was made of hydroxyethyl methacrylate was placed on her right eye in order to seal the wound.

On day 2 after the injury, the Seidel test was negative and the use of the soft contact lens was discontinued. There was no progression of cataract. However, there was an increase in cell infiltration with conjunctival injection.

On day 3, folds of Descemet membrane appeared (Fig. 2a, b). BCVA of the right eye decreased to 0.7. There was no improvement in the corneal cell infiltration. Slit-lamp examination and a repeat AS-OCT suggested inner wound closure (Fig. 2c, d). The corneal endothelial cell density was $2,778 / \mathrm{mm}^{2}$ in the right eye and $2,793 / \mathrm{mm}^{2}$ in the left eye. Steroid therapy was withheld because fungal infection had not been ruled out.

On day 9, the patient reported no ocular pain. Slight cells were observed in the anterior chamber with slight conjunctival injection. There was improvement in cell infiltration. A culture test revealed the presence of Propionibacterium acnes (P. acnes), which is sensitive to both levofloxacin and cefmenoxime. The test was negative for fungi. Because both antibiotics were thought to be effective, we continued the same treatment and decreased the frequency to 6 times a day. Atropine and acetazolamide were discontinued.

On day 14, BCVA of the right eye had improved to 0.9 . The opacification of the anterior lens capsule decreased with slight cells in the anterior chamber. However, cell infiltration at the deep corneal stroma persisted.

On day 26, the patient was completely asymptomatic. The opacification and cell infiltration at the center of the cornea had improved (Fig. 3a, b). AS-OCT showed healing of the corneal wound with reduction in the central corneal thickness to $523 \mu \mathrm{m}$ (Fig. 3c). The corneal shape analysis suggested that the wound did not affect corneal irregular astigmatism (Fig. 3d). No progress of cataract was observed and BCVA improved to 1.0.

\section{Discussion}

In the current case, the chestnut burr caused corneal tear with damage to the iris and lens capsule as well. As per the ocular trauma classifications, this was an open globe injury (zone 1) [4]. Slit-lamp examination did not reveal any intraocular foreign bodies. Clinical and AS-OCT examination of the corneal full-thickness tear were suggestive of penetration of the cornea by the sharp needle of the chestnut burr, rather than injury resulting from the impact of the chestnut fruit.

At first visit, a surgical suture was considered for sealing the wound. However, a suture would have induced irregular astigmatism because the wound was in the center of the cornea. Thus, anticipating a self-sealing wound, we used a soft contact lens and it was effective. A soft contact lens may be useful if a corneal wound is small and sharp with adequately maintained anterior chamber depth.

In this case, antibiotic treatment was performed because cell infiltration of the corneal wound and conjunctival injection were observed. On day 3, cell infiltration worsened and folds of Descemet membrane appeared. Additionally, the corneal thickness increased. There was better penetration of the antibiotics when the corneal wound was open, and the penetration may have reduced after the wound closed. We performed continual antibiotic instillation frequently, and cell infiltration improved. Although bacterial examination of the corneal scrapings showed gram-positive cocci, the culture test result was positive for $P$. acnes, demonstrating gram-positive rods. Gram-positive cocci were clinically thought to be the 
main causative organism. Therefore, contamination by P. acnes may have been the cause of the discrepancy.

AS-OCT showed corneal wound closure and improvement in the corneal edema with treatment. Furthermore, it supplied information on accurate corneal thickness and anterior chamber depth. AS-OCT was a valuable tool to noninvasively observe wound shape and detect the presence of intracorneal foreign bodies, as reported previously [5].

Ocular trauma by plant material can predispose patients to fungal infection. In particular, it has been pointed out that fungal keratitis may be caused by soil [6]. In the current case, cell infiltration in the deep corneal stroma was observed, which was responsive to topical antibiotics. Although bacterial keratitis was suspected, fungal infection could not be ruled out. Hence, topical steroids were withheld during treatment. We planned to institute antifungal treatment if the ulcer was unresponsive to antibiotics. It is well known that traumatic cataract progresses after lens injury. In the current case, the chestnut burr breached the anterior capsule of the lens through the cornea. A follow-up examination 1 month after trauma did not reveal any progress of the cataract. However, long-term observation might be necessary.

We report a case of ocular trauma from a chestnut burr that penetrated the cornea and reached the anterior capsule of the lens. This study demonstrated that AS-OCT is a useful tool to examine corneal wounds in order to determine proper treatment.

\section{Statement of Ethics}

This study adhered to the principles of the declaration of Helsinki. The patient provided written informed consent for the treatment.

\section{Disclosure Statement}

None of the authors have any conflicts of interest related to this submission.

\section{References}

1 Garcia-Garcia GP, Esaa-Caride NC, Jurado-Guano ND, Munoz-Bellido L: Ocular injury with chestnut burr: our experience. Cornea 2016;35:1315-1319.

-2 Zhang ZD, Huang MK, Zhou R, Qu J: A 7-year retrospective study for clinical features and visual outcome of chestnut burr-related ocular injuries. Graefes Arch Clin Exp Ophthalmol 2013;251:1247-1249. Joseph T: Paddy grain injury of the eye. A study of 277 cases. Br J Ophthalmol 1968;52:191-197. Pieramici DJ, Sternberg P Jr, Aaberg TM Sr, Bridges WZ Jr, Capone A Jr, Cardillo JA, de Juan E Jr, Kuhn F, Meredith TA, Mieler WF, Olsen TW, Rubsamen P, Stout T: A system for classifying mechanical injuries of the eye (globe). The Ocular Trauma Classification Group. Am J Ophthalmol 1997;123:820-831.

-5 Celebi AR, Kilavuzoglu AE, Altiparmak UE, Cosar CB, Ozkiris A: The role of anterior segment optical coherence tomography in the management of an intra-corneal foreign body. Springerplus 2016;5:1559.

6 Uno T: Ocular mycosis (in Japanese). Nihon Ishinkin Gakkai Zasshi 2008;49:175-179. 


\section{Case Reports in Ophthalmology}
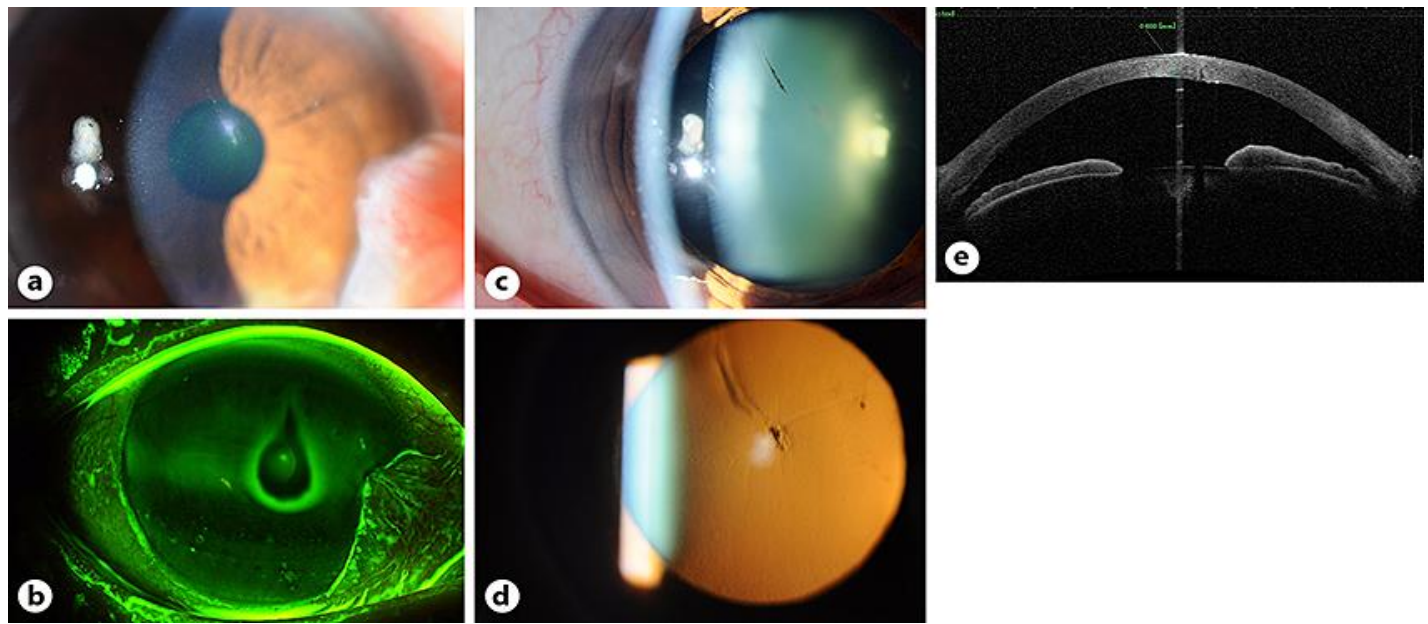

Fig. 1. Clinical images of the patient at first visit. a Slit-lamp examination showed a perforation at the center of the cornea. $\mathbf{b}$ The Seidel test was positive with fluorescein staining. c Pigments were attached on the anterior lens capsule. $\mathbf{d}$ A retroillumination image showed that the anterior capsule was opacified. e An ASOCT image showed a corneal perforation that reached the corneal endothelial layer.
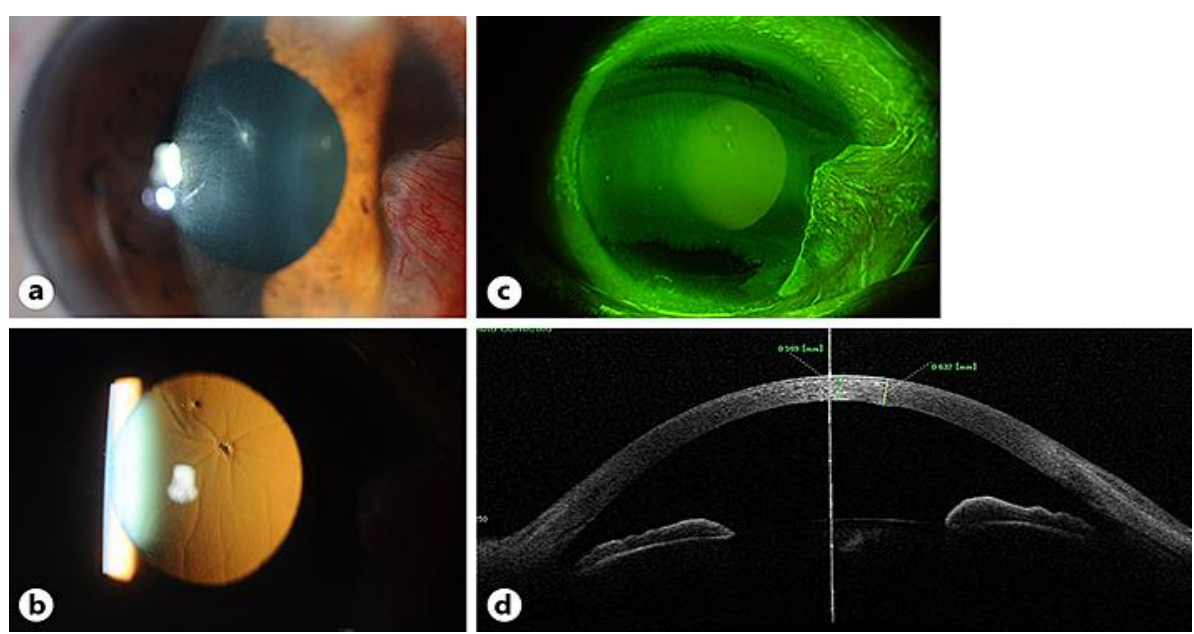

Fig. 2. Clinical images of the patient on day 3. a Slit-lamp examination showed that folds of Descemet membrane appeared with cell infiltration. b A retroillumination image showed folds of Descemet membrane. c The Seidel test was negative. d AS-OCT image showed that the wound had closed and the endothelial layer had become smooth. 


\section{Case Reports in Ophthalmology}

\begin{tabular}{l|l}
\hline Case Rep Ophthalmol 2018;9:154-159 \\
\hline DOI: 10.1159/000487076 & $\begin{array}{l}\text { @ 2018 The Author(s). Published by S. Karger AG, Basel } \\
\text { www.karger.com/cop }\end{array}$ \\
\hline
\end{tabular}

Ono et al:: Optical Coherence Tomography Examination of the Anterior Segment in a Case of Corneal Perforation and Lens Trauma by Chestnut Burr
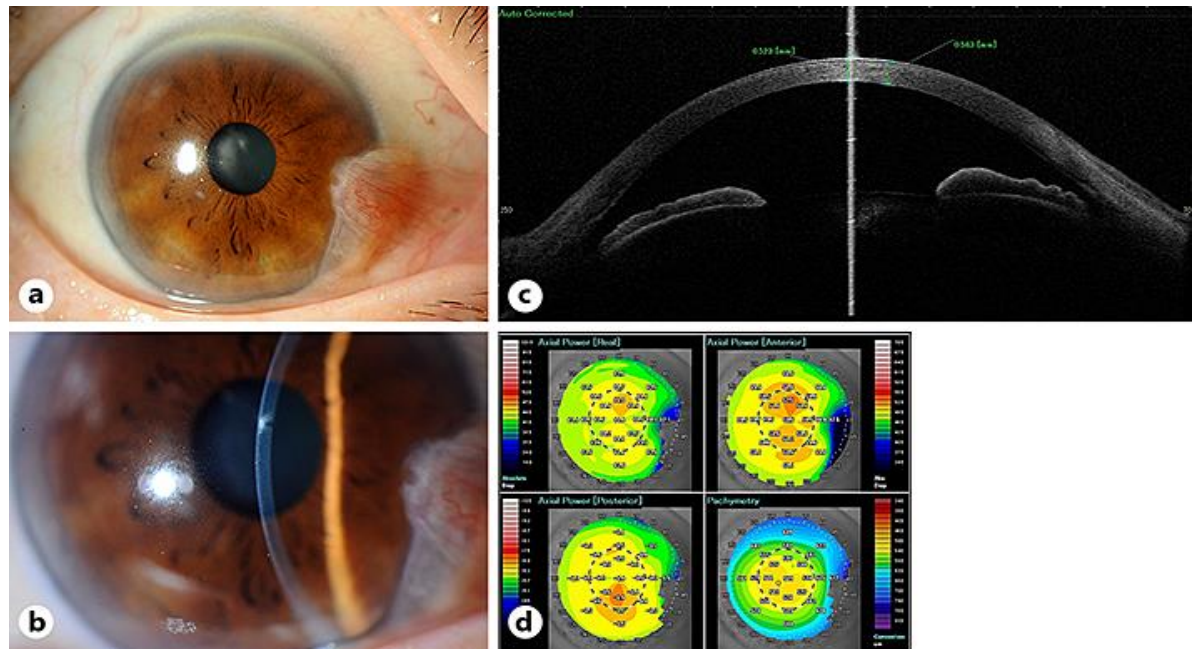

Fig. 3. Clinical images of the patient on day 26. a Slit-lamp examination showed that cell infiltration had disappeared. b Slight opacification was left at the center of cornea. c AS-OCT showed that the wound had closed and corneal edema had improved. $\mathbf{d}$ AS-OCT analysis suggested that the corneal irregularity caused by the wound was small. 